\title{
Dynamic Measurements on Polymer Liquid Crystals- Aqueous Solutions of Hydroxypropyl Cellulose
}

\author{
by \\ Masao Horio*1, Ei-ichi KAMEI*2, and Hisataka UCHIMURA*2 \\ *1Department of Polymer Chemistry, Faculty of Engineering, \\ Kyoto University, Sakyoku, Kyoto 606 and \\ ${ }^{*}$ Plastics Research Laboratory, Ube Industries, Ltd., \\ 3-10 Nakamiyakitacho, Hirakata, Osaka 573
}

(Received : June 20, 1984)

\begin{abstract}
Dynamic measurements are made with cone and plate geometry on the aqueous solutions of hydroxypropyl cellulose (HPC) in the frequency region from $10^{-1}$ to $10^{2} \mathrm{radian} \cdot \mathrm{sec}^{-1}$. The torque resulting from sinusoidally alternating strain imposed on the cholesteric phase of $60 \mathrm{wt} \%$ HPC solution exhibits undeformed sinusoidal wave which is out of phase with the input strain, as is the case with linear viscoelastic materials. Distinct difference in dynamic properties is recognized between optically isotropic and optically anisotropic solutions of HPC. The critical concentration which marks the transition from isotropic to anisotropic state is about $42 \mathrm{wt} \%$ at $25^{\circ} \mathrm{C}$ at which most of the experiments are made. The logarithmic plots of dynamic storage modulus $\left(G^{\prime}\right)$ and loss modulus $\left(G^{\prime \prime}\right)$ against angular frequency for the optically anisotropic solutions of HPC tend to form a plateau in the low frequency region, while the plots for the optically isotropic solutions decline steeply with decreasing frequency, as is usual for the ordinary polymer solutions and melts. The order of $G^{\prime}$ and $G^{\prime \prime}$ of liquid crystalline solutions expressed in terms of concentration is $60>45>48>50 \mathrm{wt} \%$. A minimum value is found at a concentration of about $50 \mathrm{wt} \%$ at which the solution first exhibits the cholesteric texture and iridescence. The effect of strain amplitude upon $G^{\prime}$ and $G^{\prime \prime}$ of cholesteric phase is minor, as in the case of linear viscoelastic materials. The real component $\eta^{\prime}$ of complex viscosities of the optically anisotropic solutions tends to increase with decreasing angular frequency, while the values of $\eta^{\prime}$ of the optically isotropic solutions are constant in the low frequency region. The plot of $\eta^{\prime}$ against concentration shows a sharp peak at about 42 wt $\%$ at which the transition takes place and a minimum point at about $50 \mathrm{wt} \%$ at which the cholesteric structure is formed. The difference between $\eta^{\prime}$ and the steady-shear viscosity $\eta$ is interpreted in terms of the susceptibility of cholesteric phase to the prolonged effect of shear. The HPC solution containing $\mathrm{TiO}_{2}$ particles shows the dynamic properties similar to those of liquid crystalline solutions, but the resemblance seems to be casual. The stress relaxation taking place after the cessation of steady flow is measured. The later stage of relaxation of $60 \mathrm{wt} \%$ solution at $23^{\circ} \mathrm{C}$ is explained in terms of a Maxwell model with a relaxation time of $42 \mathrm{sec}$. Most of the relaxation takes

*1 To whom all the correspondence should be addressed. 堀尾正雄: 京都大学工学部高分子化学教室 $\mathbf{T} 606$ 京都市左京区吉田本町

*2 亀井衛一, 内村寿孝: 宇部興産(株)枚方樹脂研究所 厂573 枚方市中宮北町 3-10
\end{abstract}


place within $5 \mathrm{sec}$. This early stage of relaxation is the origin of production of zigzagshaped fibrillar structure in the fibers and films made from liquid crystalline solutions. The dynamic properties of cholesteric liquid crystals of HPC are not permanent but variable with the history of mechanical treatments.

\section{INTRODUCTION}

The monograph on rheology of liquid crystals by Porter and Johnson $^{1)}$ published in 1967 drew attention to the new field of rheological science. Many original papers and monographs in this stimulating area have appeared so far in various journals. Most recently Asada and Onogi ${ }^{2}$ ) reviewed the papers on the rheological properties of polymer liquid crystals focusing interest on their own rheo-optical studies. A general survey of the papers thus far published leads to the discovery of fact that the study on the dynamic properties of liquid crystals is surprisingly scanty. In the course of the study being made of the formation of fibers and films from various polymer liquid crystals, the author $(\mathrm{H})$ found that the viscoelastic behavior of liquid crystals played an important role in the generation of characteristic morphology of zigzag-shaped fibrils in the products $^{3) \sim 5)}$. This induced us to make the dynamic measurements on polymer liquid crystals. In this paper, the dynamic properties of aqueous solutions of hydroxypropyl cellulose* are treated. The optically anisotropic solutions show markedly different dynamic properties from those of the optically isotropic solutions. Reference is made particularly to the viscoelastic behavior of suspensions in view of the apparent resemblance of their dynamic properties to those of the liquid crystals.

\section{MATERIAL AND APPARATUS}

\section{Hydroxypropyl cellulose}

The commercial product, HPC-SL of Nisso Co. (henceforth simply HPC), was used as delivered. The thin layer chromatogram demonstrates that the product is not contaminated with isolated poly(propylene oxide). The ash content is $0.27 \%$. The average molecular weights determined by the GPC data are, $\bar{M}_{\mathrm{w}}=104,300, \bar{M}_{\mathrm{n}}=41,600$ and $\bar{M}_{\mathrm{w}} / \bar{M}_{\mathrm{n}}=2.5$. The molar substitution (average number of hydroxypropyl groups per anhydroglucose unit) determined from the ${ }^{1} \mathrm{H}-\mathrm{NMR}$ spectrum by the method of Ho et al.6) is 3.5. The degree of substitution (average number of hydroxyl groups substituted per anhydroglucose unit) determined from the ${ }^{13} \mathrm{C}-\mathrm{NMR}$ spectrum by the method of Kimura et al. ${ }^{7)}$ is 2.3. Calcd. for $\mathrm{C}_{16.5} \mathrm{H}_{31.0} \mathrm{O}_{8.5}: \mathrm{C}$ $54.23, \mathrm{H} 8.55$; Found : C 53.83, H 8.57.

HPC is soluble in water. The solution with a concentration of $39 \mathrm{wt} \%$ or below is clear and optically isotropic at $18^{\circ} \mathrm{C}$. On the other hand, the solution with a concentration of 40 wt $\%$ or above is turbid and optically anisotropic. Therefore,

* O-(2-hydroxypropyl) cellulose. the critical concentration that marks the transition from isotropic to anisotropic state is about $40 \mathrm{wt} \%$ at $\left.18^{\circ} \mathrm{C} .{ }^{5}\right)$ The critical concentration depends on temperature. At $25^{\circ} \mathrm{C}$, at which most of the experiments were conducted, the critical concentration is about $42 \mathrm{wt} \%$. The solutions whose concentrations are higher than about $50 \mathrm{wt} \%$ show at room temperature the iridescence characteristic of cholesteric liquid crystals.4),5)

\section{Apparatus}

Rheometrics Mechanical Spectrometer RMS-605 was used with cone and plate geometry. The diameters of cone and plate were $50 \mathrm{~mm}$. The angle between cone and plate was 0.04 radian. For the dynamic measurements, sinusoidally varying angular displacement was imposed on the surface of cone in contact with sample solution and the resulting torque acting on the surface of plate was measured by a transducer. Various viscoelastic quantities were calculated from the torque and phase angle by a computer and the results were automatically recorded on a $\log -\log$ graph paper as a function of angular frequency which covered the range from $10^{-1}$ to $10^{2}$ radian $\cdot \mathrm{sec}^{-1}$. The strain amplitude, expressed by the percentage of oscillation amplitude to the cone angle, both given in radian, was about $100 \%$ unless otherwise noted. The effect of strain on the viscoelastic quantities was studied by an additional series of experiments done with strain amplitudes of about 10 , 25,50 and $100 \%$. For the steady-shear tests, the cone was rotated at a constant angular speed and the torque produced on the surface of plate was measured. The shear rate was varied in the range from $10^{-1}$ to $10^{2} \mathrm{sec}^{-1}$.

\section{RESULTS}

\section{Linear Viscoelasticity}

Because the viscoelastic quantities referred to in this study are described by the linear constitutive equations, the thing that must be known first is whether the liquid crystalline materials under study exhibit the linear viscoelastic behavior in the dynamic measurements. If the behavior is linear viscoelastic, both strain and stress in the dynamic experiments will alternate sinusoidally with a certain phase angle between them. This relationship was examined at $23^{\circ} \mathrm{C}$ on an optically isotropic solution with a HPC content of $40 \mathrm{wt} \%$ and an optically anisotropic solution with a HPC content of $60 \mathrm{wt} \%$ known as a cholesteric liquid crystal. In the experiments, the sinusoidal angular displacement with a strain amplitude of $100 \%$ was imposed on the surface of cone in contact with solution and the torque detected on the surface of plate was recorded on a graph as a function of time. The frequency was varied from 
0.1 to $1 \mathrm{radian} \cdot \mathrm{sec}^{-1}$. Fig. 1 shows the automatic recording of the input strain $(\gamma)$ and the output torque $(M)$ at the sinusoidal oscillation of cone with a frequency of 0.32 radian. $\mathrm{sec}^{-1}$. It is found that the torque which is in phase with the shear stress* alternates sinusoidally without any substantial deformation of curve and is out of phase with the strain. The result demonstrates, therefore, that the solution is practically linear viscoelastic. The experiments made with frequencies of $0.1,0.64$ and 1 radian $\cdot \mathrm{sec}^{-1}$ gave the similar results showing the linear viscoelastic behavior of sample.

Fig. 2 shows the automatic recording of the wave trains of the input strain $(\gamma)$ and the output torque $(M)$ for the

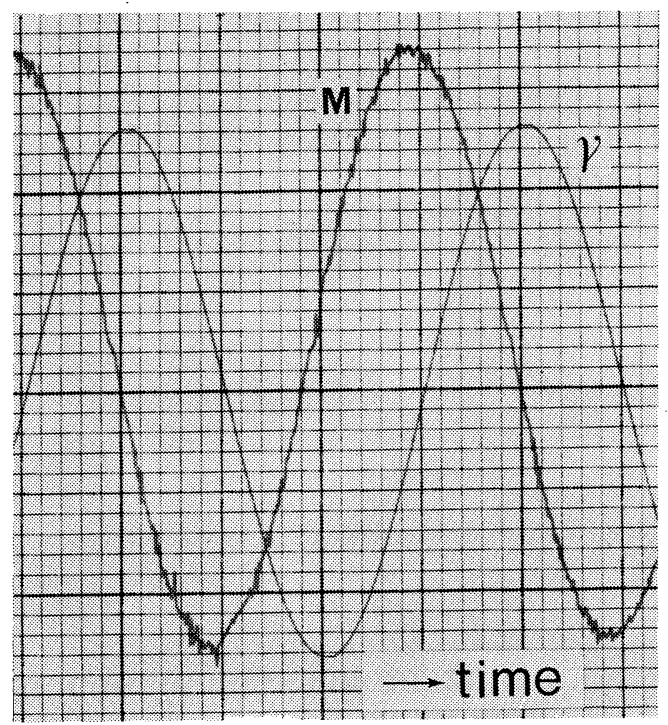

Fig. 1 Automatic recording of input sinusoidal strain $(\gamma)$ and output torque $(M)$ for $40 \mathrm{wt} \%$ solution of HPC. Angular frequency, $0.32 \mathrm{radian} \cdot \mathrm{sec}^{-1}$.

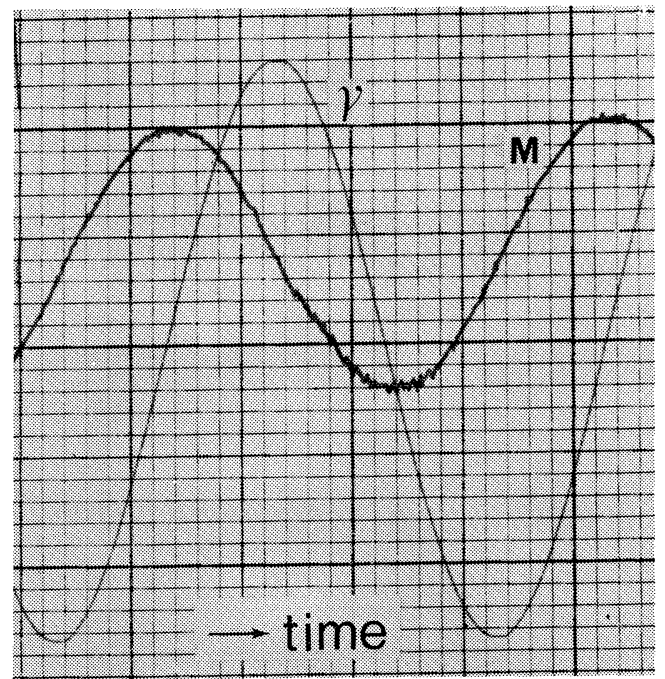

Fig. 2 Automatic recording of input sinusoidal strain $(\gamma)$ and output torque $(\boldsymbol{M})$ for $60 \mathrm{wt} \%$ solution of HPC. Angular frequency, $0.32 \mathrm{radian} \cdot \mathrm{sec}^{-1}$.

* The shear stress is given by $3 M / 2 \pi R^{3}$, where $M$ is the torque and $R$ is the radius of cone and plate. cholesteric liquid crystal of HPC with a concentration of 60 wt $\%$ in the sinusoidal oscillation with a frequency of 0.32 radian $\cdot \mathrm{sec}^{-1}$. Here also, both strain and torque alternate sinusoidally with a certain difference in phase and no perceptible distortion is found on the curve of output torque. At other frequencies also, the similar relationship is recognized between strain and torque. This experiment may be employed as a criterion for the linear viscoelasticity of the liquid crystal. The insensitiveness of the viscoelartic quantities to strain amplitude will be described later (see Fig. 7).

\section{Dynamic Modulus}

The dynamic measurement on any linear viscoelastic system at a given frequency provides simultaneously two quantities, i.e., the storage shear modulus $G^{\prime}$ and the loss shear modulus $G^{\prime \prime}$ which are determined from the torque and phase angle on the basis of the equations, $G^{\prime}=\left(\sigma_{0} / \gamma_{0}\right) \cos \delta$ and $G^{\prime \prime}=\left(\sigma_{0} / \gamma_{0}\right)$ $\sin \delta$, where $\sigma_{0}$ and $\gamma_{0}$ are the amplitudes of stress and strain, respectively, and $\delta$ is a phase angle between strain and stress.

The measurements of $G^{\prime}$ and $G^{\prime \prime}$ reveal the distinct difference in dynamic properties between the liquid crystals and the optically isotropic solutions. Fig. 3 shows the logarithmic plots of $G^{\prime}$ and $G^{\prime \prime}$ against angular frequency $\omega$ for an optically isotropic solution of HPC with a concentration of $39 \mathrm{wt} \%$ at $25^{\circ} \mathrm{C}$. In the frequency region covered, the curves of $G^{\prime}$ and $G^{\prime \prime}$ are apparently similar in shape to those of the ordinary polymer solutions. $G^{\prime}$ decreases rapidly with decrease in frequency and the $\log$ - $\log$ plot of $G^{\prime}$ vs. $\omega$ shows a straight line with a slope of 2 in the region of low frequencies. In the high frequency region, the curve shows a tendency to flatten. The curve of $G^{\prime \prime}$, on the other hand, shows a straight line with a slope of unity in the region of low frequencies and flattens in the high frequency region like the curve of $G^{\prime}$. It is interesting to notice that the molecular-theoretical formulations of $G^{\prime}$ and $G^{\prime \prime}$ of the dilute solutions containing rigid macromolecules ${ }^{8), 9)}$ result in the curves of $G^{\prime}$ and $G^{\prime \prime}$ which have the similar characteristic feature to that of the curves in Fig. 3.

In contrast to the optically isotropic solution described above, the optically anisotropic solution with a high content of HPC exhibits the considerably different dynamic properties. Fig. 4 shows the logarithmic plots of $G^{\prime}$ and $G^{\prime \prime}$ vs. $\omega$ for $60 \mathrm{wt} \%$ HPC solution forming the cholesteric liquid crystal. Contrary to Fig. 3, the curves of $G^{\prime}$ and $G^{\prime \prime}$ of the liquid crystal do not decline steeply in the low frequency region but flatten showing a tendency to form a plateau. In the high frequency region up to $10^{2} \mathrm{radian} \cdot \mathrm{sec}^{-1}$, both $G^{\prime}$ and $G^{\prime \prime}$ plotted in the logarithmic scale increase linearly with frequency without showing a tendency to flatten. It is particularly important to notice that the abnormal dynamic properties of liquid crystals appear most prominently in the region of low frequencies.

Any solution whose HPC content is higher than the critical concentration shows the $G^{\prime}$ and $G^{\prime \prime}$ curves which flatten in the 


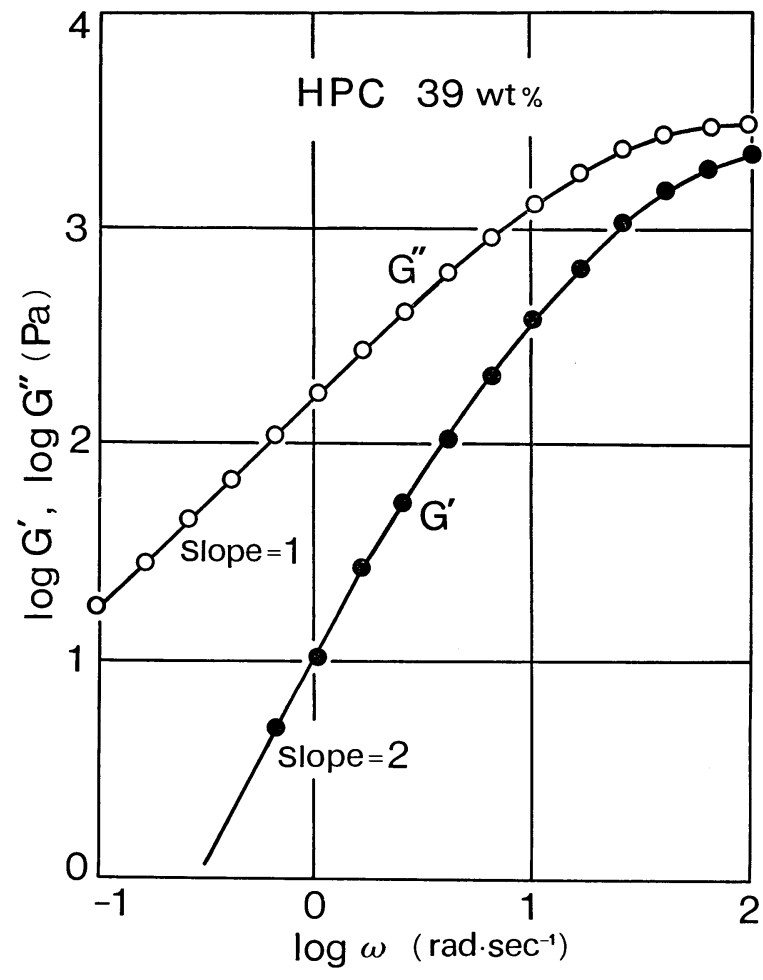

Fig. 3 Logarithmic plots of storage and loss shear moduli of $39 \mathrm{wt} \%$ solution of $\mathrm{HPC}$ at $25^{\circ} \mathrm{C}$ against angular frequency.

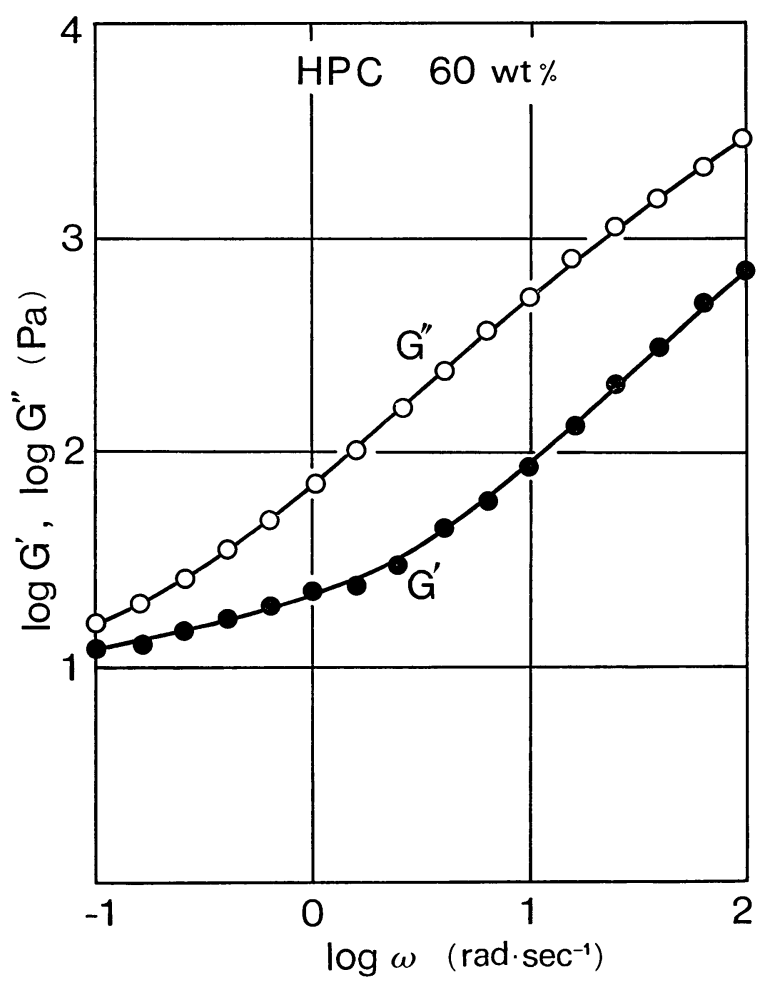

Fig. 4 Logarithmic plots of storage and loss shear moduli of $60 \mathrm{wt} \%$ solution of $\mathrm{HPC}$ at $25^{\circ} \mathrm{C}$ against angular frequency. low frequency region. Fig. 5 shows the $\log G^{\prime}$ vs. $\log \omega$ curves of the optically anisotropic solutions with HPC contents of $45,48,50$ and $60 \mathrm{wt} \%$ in comparison with the curves of the optically isotropic solutions with HPC contents of 39 and $41 \mathrm{wt} \%$. As a result of flattening of $G^{\prime}$ curves, the liquid crystalline solutions show in the low frequency region the larger values of $G^{\prime}$ than those of the optically isotropic solutions. In the high frequency region, on the other hand, the isotropic solutions exhibit the larger values of $G^{\prime}$ than those of the liquid crystalline solutions. Of particular interest is the marked difference between 41 and $45 \mathrm{wt} \%$ solutions; $41 \mathrm{wt} \%$ is just below the critical concentration (about $42 \mathrm{wt} \%$ ), while 45 wt $\%$ is a little above the critical concentration. It is found, therefore, that the critical concentration draws a sharp line of demarcation between the two different types of dynamic properties.

The relation between $G^{\prime}$ and concentration of liquid crystalline solution is not simple, as can be seen in Fig. 5. The magnitude of $G^{\prime}$ of solution is not in regular order of concentration. The values of $G^{\prime}$ in the low frequency region can be written in decreasing order in terms of concentration as follows: $60>45$ $>48>50 \mathrm{wt} \%$. The value of $G^{\prime}$ decreases with increase in concentration up to $50 \mathrm{wt} \%$ and then begins to rise. Namely, the minimum value of $G^{\prime}$ is reached at around $50 \mathrm{wt} \%$. The concentration of $50 \mathrm{wt} \%$ can be designated as another critical concentration, because at this concentration the solution first

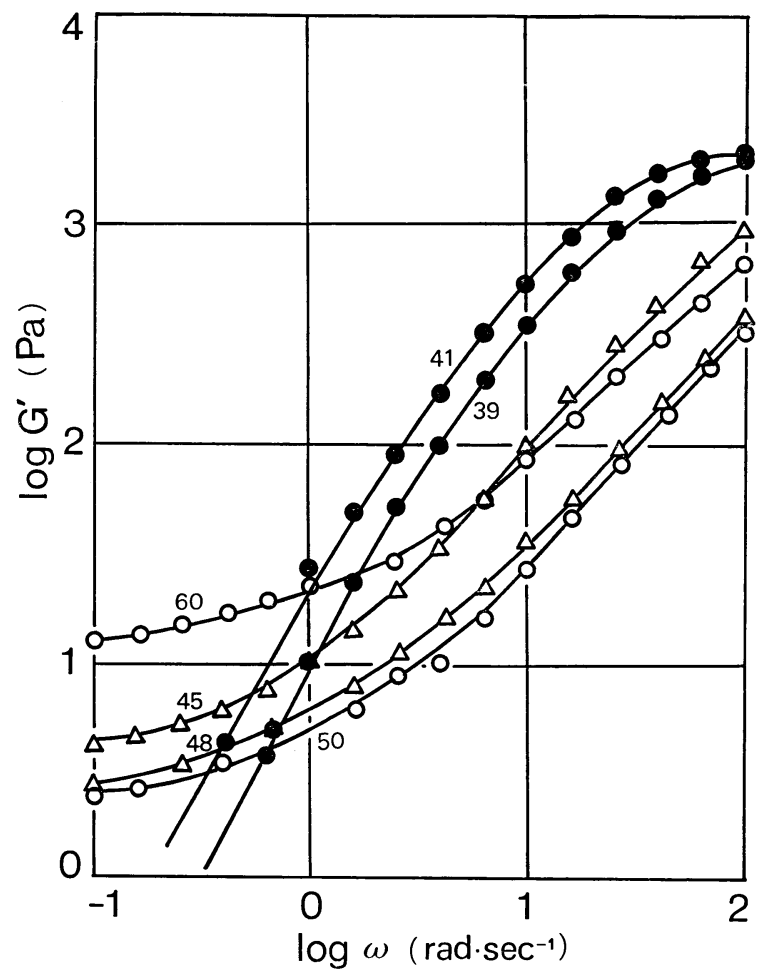

Fig. 5 Logarithmic plots of storage shear moduli of 39 , $41,45,48,50$ and $60 \mathrm{wt} \%$ solutions of HPC at $25^{\circ} \mathrm{C}$ agair $\mathbf{s}$ : angular frequency. 
exhibits the characteristic texture of cholesteric liquid crystal and displays the noticeable iridescence. It is interesting to see, therefore, that the state and structure of solution are faithfully reflected in the dynamic properties.

The loss shear moduli of the solutions with various HPC contents are plotted logarithmically against angular frequency in Fig. 6. The curves of $G^{\prime \prime}$ of the optically anisotropic solutions tend to flatten in the low frequency region contrary to the curves of the optically isotropic solutions which decline linearly with a slope of unity. In agreement with the case of $G^{\prime}$, the values of $G^{\prime \prime}$ in the low frequency region expressed in terms of concentration of solution can be arranged in decreasing order of magnitude as follows: $60>45>48>50 \mathrm{wt} \%$. The minimum value of $G^{\prime \prime}$ is shown by $50 \mathrm{wt} \%$ solution, as in the case of $G^{\prime}$.

A series of experiments was made with strain amplitudes of $10,25,50$ and $100 \%$ for the examination of the effect of strain on $G^{\prime}$ and $G^{\prime \prime}$. For the optically isotropic solution with a HPC content of $40 \mathrm{wt} \%$, there was no effect of strain on the dynamic behavior of solution, as was expected. For the liquid crystalline solutions having their own characteristic structures, a little different result was obtained. Fig. 7 shows the logarithmic plots of $G^{\prime}$ and $G^{\prime \prime}$ against angular frequency for the liquid crystalline solution with a HPC content of $60 \mathrm{wt} \%$ measured with strain amplitudes of 10 and $100 \%$. The curves of $G^{\prime}$ and $G^{\prime \prime}$ obtained with 25 and 50\% amplitudes lie between

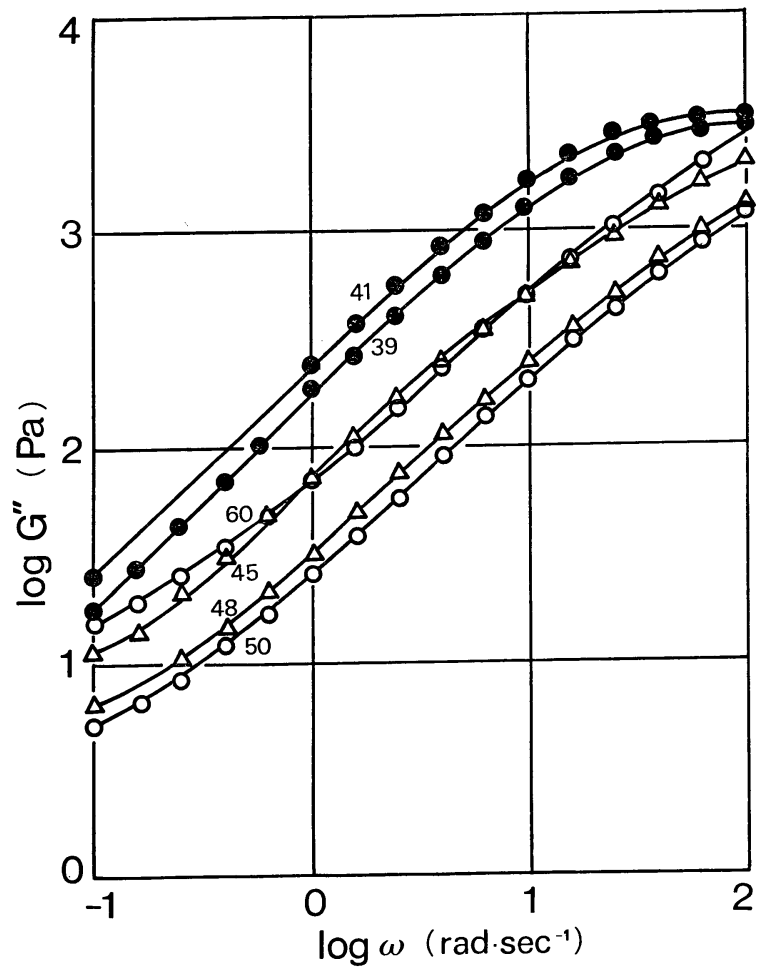

Fig. 6 Logarithmic plots of loss shear moduli of 39,41 , $45,48,50$ and $60 \mathrm{wt} \%$ solutions of $\mathrm{HPC}$ at $25^{\circ} \mathrm{C}$ against angular frequency.

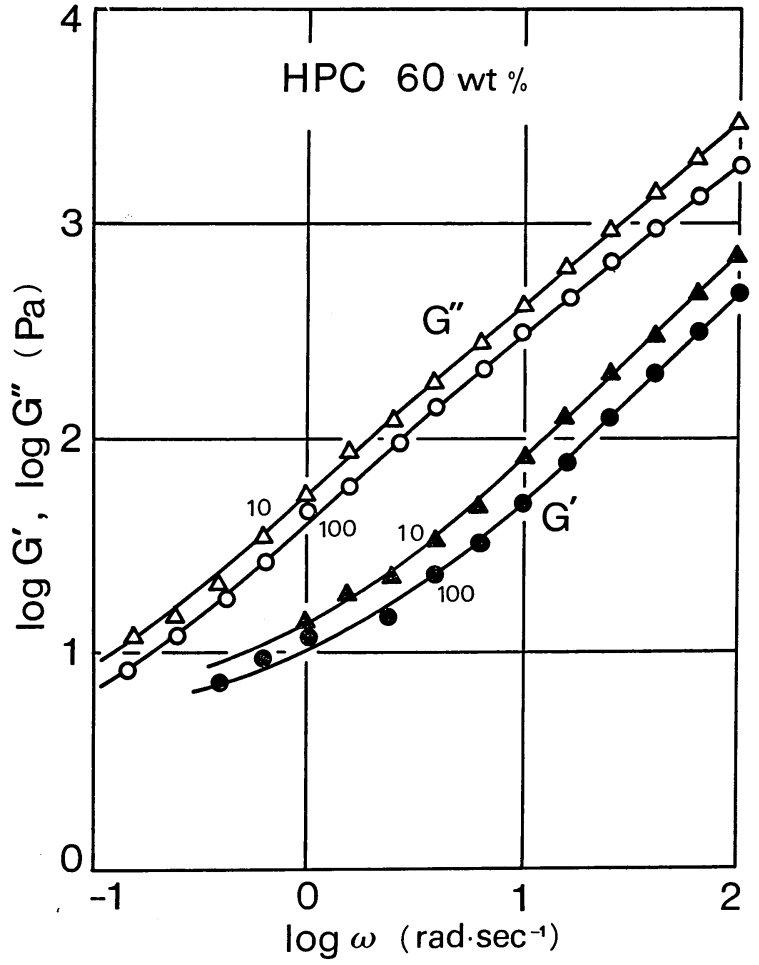

Fig. 7 Logarithmic plots of $G^{\prime}$ and $G^{\prime \prime}$ against angular frequency for $60 \mathrm{wt} \%$ solution of HPC at strain amplitudes of 10 and $100 \%$.

the curves of 10 and $100 \%$ amplitudes in a regular sequence showing that the magnitudes of $G^{\prime}$ and $G^{\prime \prime}$ tend to decrease with increase in amplitude. The experiments suggest that the forced oscillation produces some effect on the structure of the liquid crystal, but, as can be deduced from Fig. 7, the effect of strain on $G^{\prime}$ and $G^{\prime \prime}$ is just small when the strain amplitude is less than $100 \%$. Therefore, the viscoelastic behavior of the liquid crystal under discussion may be regarded as linear under the experimental conditions of the dynamic measurements in this study. This means that the structure of liquid crystal is disturbed only slightly, if any, by the angular oscillation. As a matter of fact, the sample oscillating with a strain amplitude of $100 \%$ at various frequencies displays iridescence in quite a similar manner as in the quiescent state.

\section{Dynamic Viscosity}

The real part of complex viscosity, $\eta^{\prime}$, which is equal to $G^{\prime \prime} / \omega$ in the linear viscoelastic system is shown in Fig. 8 as a function of angular frequency in doubly logarithmic scale. Here also, a marked difference is seen between optically isotropic solutions and optically anisotropic solutions. The optically isotropic solutions with HPC contents of 39 and 41 wt $\%$ show the Newtonian behavior in the low frequency region, in which $\eta^{\prime}$ is constant independent of frequency. On account of this behavior, the low-frequency limit value of $\eta^{\prime}$ can be definitely estimated. In the high frequency region, $\eta^{\prime}$ decreases gradually with frequency as is usual for the ordinary 


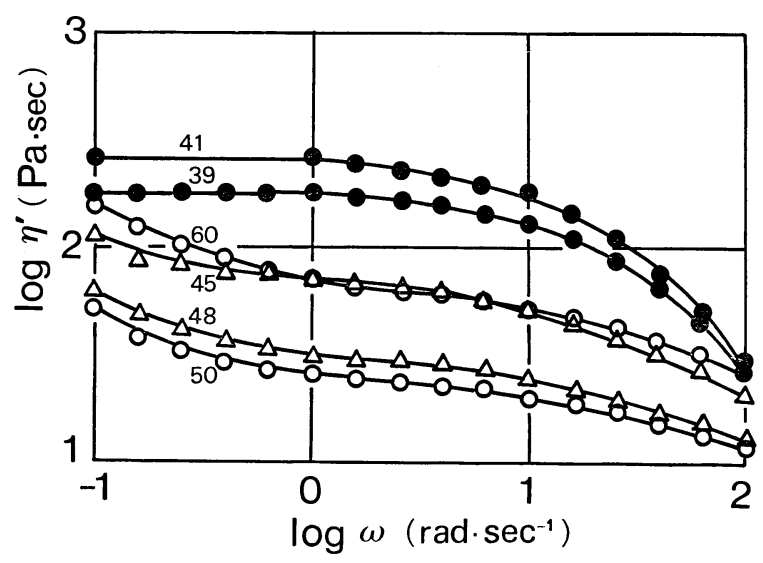

Fig. 8 Logarithmic plot of real part of complex viscosity against angular frequency for $39,41,45,48,50$ and $60 \mathrm{wt} \%$ solutions of $\mathrm{HPC}$ at $25^{\circ} \mathrm{C}$.

polymer solutions.

The optically anisotropic solutions behave dynamically in a different manner. The $\log \eta^{\prime}$ vs. $\log \omega$ curves of the optically anisotropic solutions can be divided in three parts with respect to frequency region. In the low frequency region, the curves turn upwards to the left, in the middle frequency region, the curves are almost parallel and tend towards horizontal and in the high frequency region, the curves turn downwards to the right. This characteristic relationship between dynamic viscosity and frequency is very similar to the relationship between steady-flow viscosity and shear rate of various liquid crystals pointed out by Asada et al. ${ }^{2), 22)}$

As was the case with $G^{\prime}$ and $G^{\prime \prime}$, the relationship between $\eta^{\prime}$ and HPC content of liquid crystalline solution is not simple. Because the liquid crystals have no definite low-frequency limit value of $\eta^{\prime}$, the values in the middle frequency region where the curves of $\eta^{\prime}$ are almost parallel are used for comparison. The sequence of the magnitude of $\eta^{\prime}$ at $\log \omega=0.6$ expressed in terms of concentration is $60=45>48>50 \mathrm{wt} \%$. This order of $\eta^{\prime}$ is similar to those observed with $G^{\prime}$ and $G^{\prime \prime}$. In Fig. 9, the values of $\eta^{\prime}$ at a frequency of $\log \omega=0.6$ are plotted against concentration of solution. The curve of $\eta^{\prime}$ shows a peak at a concentration of about $42 \mathrm{wt} \%$ at which the transition from the optically isotropic state to the optically anisotropic state takes place, and a minimum value of $\eta^{\prime}$ is reached at a concentration of about $50 \mathrm{wt} \%$ at which first the solution exhibits the cholesteric texture and iridescence.

\section{Steady-Flow Viscosity}

Reference to the steady-shear experiments is important in the meaning that the prolonged action of constant shear, contrary to the effect of rapidly changing oscillatory shear, brings about a radical change in the structure of solution, especially in the structure of cholesteric liquid crystal. In Fig. 10 is shown the steady-shear viscosity plotted logarithmically against shear rate for the six HPC solutions with concentrations of 39, 41, 45, 48,50 and $60 \mathrm{wt} \%$. The solutions with concentrations lower

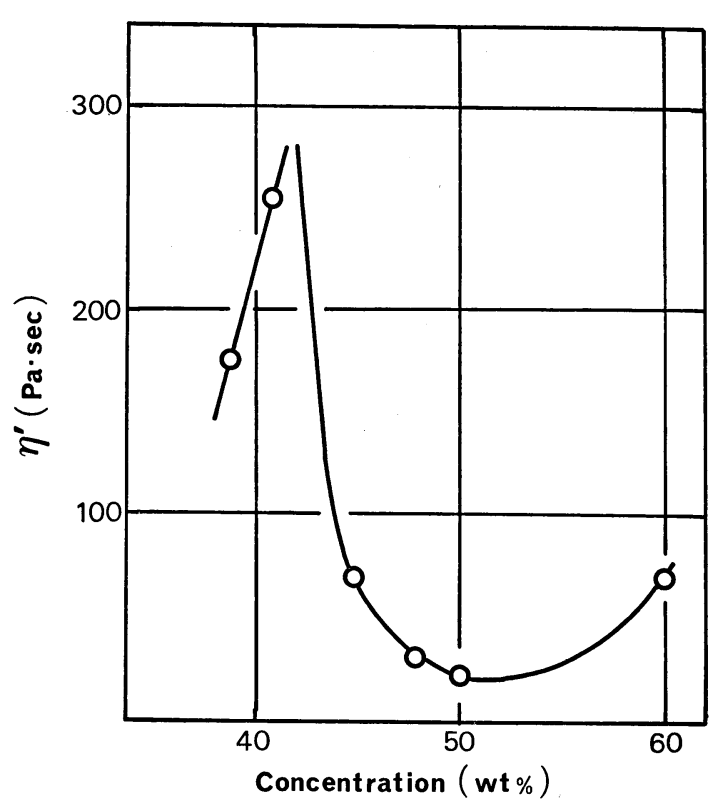

Fig. 9 Plot of real part of complex viscosities of 39,41 , $45,48,50$ and $60 \mathrm{wt} \%$ solutions of HPC at angular frequency of $\log \omega=0.6$ against concentration.

than the critical concentration (about $42 \mathrm{wt} \%$ ), such as 39 and $41 \mathrm{wt} \%$ solutions, exhibit the Newtonian behavior in the low shear rate region, as was the case with the dynamic viscosities at low angular frequencies. In the high shear rate region, the non-Newtonian flow occurs, as is usual for many other polymer solutions.

The solutions with concentrations higher than the critical concentration, on the other hand, do not show the Newtonian flow at any region of shear rate. Particularly noticeable is the behavior of 50 and 60 wt\% solutions of HPC which form the cholesteric phase in the quiescent steady state. Such solutions exhibit very high viscosities at shear rates below $1 \mathrm{sec}^{-1}$, but the viscosities decrease rapidly with increase in shear rate. In the middle region, the $\log \eta$ vs. $\log \dot{\gamma}$ curves show the

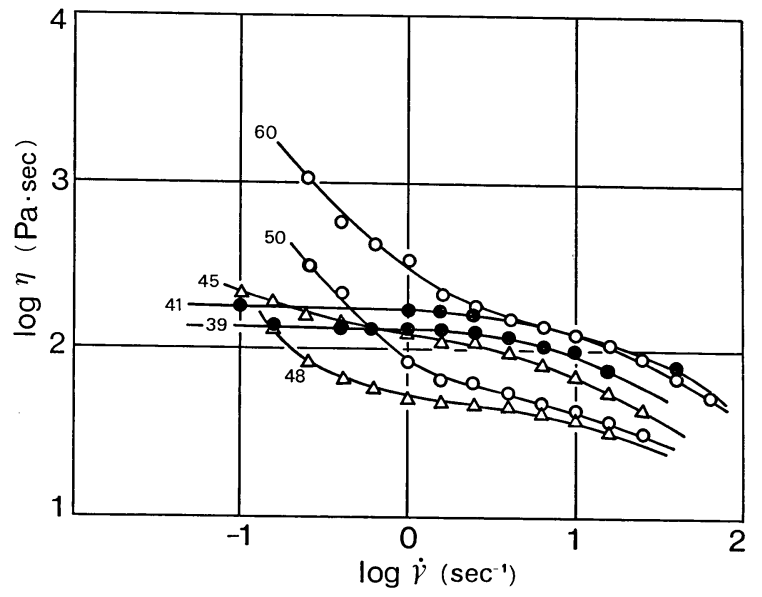

Fig. 10 Logarithmic plot of steady-shear viscosity against shear rate for $39,41,45,48,50$ and $60 \mathrm{wt} \%$ solutions of $\mathrm{HPC}$ at $25^{\circ} \mathrm{C}$. 
tendency to flatten, but do not form a plateau, continuing to decline steadily with increasing shear rate.

The marked decrease in viscosity with increasing shear rate in the region below $1 \mathrm{sec}^{-1}$ is supposed to be due to the susceptibility of cholesteric phase to shear. Although the cholesteric structure can withstand the oscillatory shear of the dynamic tests, it is highly sensitive to the prolonged shear of the steady-shear tests. Therefore, the difference between dynamic and steady-shear viscosities represented by Fig. 8 and 10, respectively, can be interpreted in terms of the difference in structure of the same solution under the different method of testing.

By the steadily imposed shear rates larger than $1 \mathrm{sec}^{-1}$, the cholesteric phase collapses to change into the nematic phase, as demonstrated first by Asada et al. ${ }^{2,22)}$ by a series of spectra of transmitted light. The exhibition of flow birefringence of solution also corroborates the same effect. ${ }^{3) \sim 5)}$ As the cholesteric phase is ruptured by degrees in proportion to shear rate, the viscosity decreases correspondingly with increasing shear rate. After the cholesteric phase is turned completely to the nematic phase at high shear rates, the liquid crystalline solutions behave like the ordinary solutions, because the molecules are arranged uniaxially in similar manner in any of the solutions. It is interesting to see that the change in structure of liquid crystalline solutions under the influence of shear rate is properly reflected in the steady-flow viscosity.

The solutions with HPC contents of 45 and $48 \mathrm{wt} \%$, which are optically anisotropic but not cholesteric, resemble the cholesteric liquid crystals in that they do not exhibit the Newtonian flow in the region of low shear rates. However, the viscosity in this region changes less prominently with shear rate in comparison with the cholesteric liquid crystals.

\section{HPC Solutions with Dispersed $\mathrm{TiO}_{2}$ Particles}

The specific dynamic properties of liquid crystalline solutions that have been described can be interpreted in terms of fine structures built up in various manners of molecular aggregation in the solutions. The texture of liquid crystalline solution of HPC differs widely according to the concentration at a given temperature. At the critical concentration or a little above, the solution under a polarizing microscope looks like a suspension with minute crystalline particles. The solution with a much higher concentration exhibits a texture made up of closely packed crystalline domains. It can be assumed that the network structure resulting from the interaction of various types of crystalline aggregates of molecules provides the characteristic relaxation mechanisms with very long time scales. These structural factors are considered to be related to the anomalous dynamic properties of liquid crystals at very low frequencies.

Of interest in this connection are the dynamic viscoelastic properties of suspensions which have been studied intensely by Onogi and coworkers ${ }^{9) \sim 18)}$ and others ${ }^{19)}{ }^{20)}$ in view of practical importance. It is pointed out in fact that the anomalous dynamic properties of suspensions at low frequencies result from various types of sturctures formed by the dispersed particles. This induced us to make up a structure in the optically isotropic solution of HPC by the addition of $\mathrm{TiO}_{2}$ particles and examine the dynamic properties in comparison with those of liquid crystalline solutions.

The isotropic solution of HPC with a concentration of 40 $\mathrm{wt} \%$ which is just below the critical concentration was used as the medium of suspension. This solution could be converted to a liquid crystal by the addition of a small quantity of HPC, but, instead of $\mathrm{HPC}, \mathrm{TiO}_{2}$ particles were added to convert the solution into suspensions. A commercial product of $\mathrm{TiO}_{2}$ (TA 500 of Fuji Chitan Kogyo, K.K.) with an average size of 0.4 $\mu \mathrm{m}$ was used as disperse particles. The concentration of suspension was expressed by the sum of weights, i.e., $40 \mathrm{wt} \%$ of $\mathrm{HPC}$ plus wt $\%$ of $\mathrm{TiO}_{2}$. The suspension of $45 \mathrm{wt} \%$, for instance, contains $40 \mathrm{wt} \%$ of $\mathrm{HPC}$ and $5 \mathrm{wt} \%$ of $\mathrm{TiO}_{2}$, and so forth. Three suspensions with concentrations of 45,50 and $60 \mathrm{wt} \%$ were prepared.

In Fig. 11 are given the logarithmic plots of $G^{\prime}$ and $G^{\prime \prime}$ against angular frequency for 45, 50 and $60 \mathrm{wt} \%$ suspensions, together with the plots for the medium of suspensions, i.e., $40 \mathrm{wt} \%$ HPC solution. The dynamic properties of 45 and 50 wt $\%$ suspensions are similar to those of the medium with no

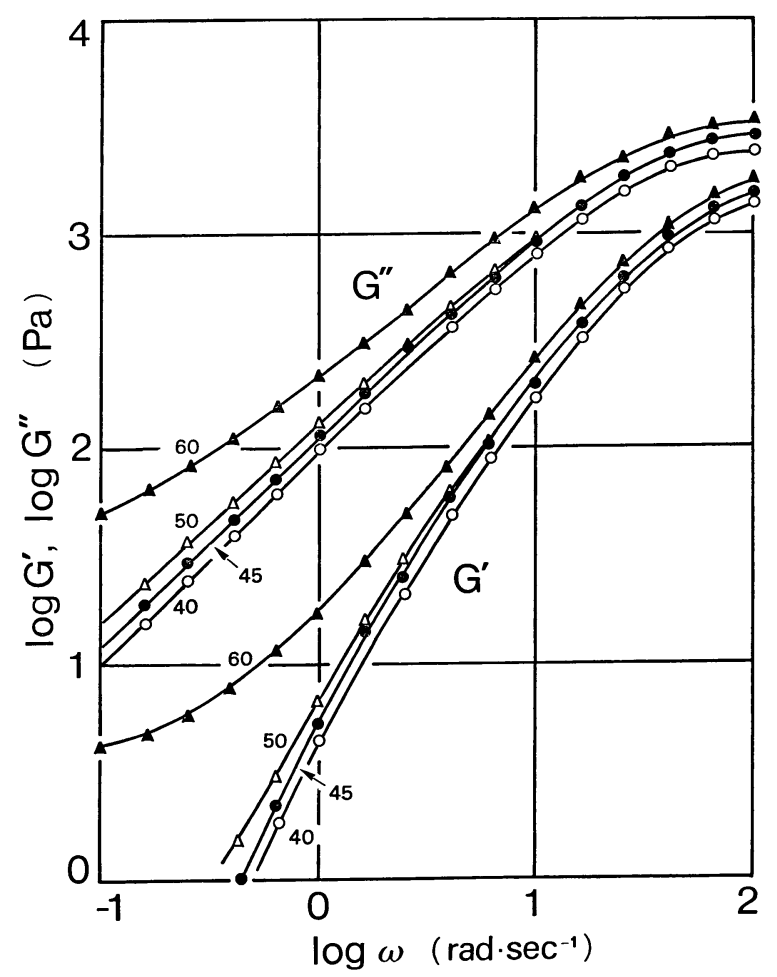

Fig. 11 Logarithmic plots of $G^{\prime}$ and $G^{\prime \prime}$ against $\omega$ for 45 , 50 and 60 wt $\%$ suspensions containing $\mathrm{TiO}_{2}$ particles in $40 \mathrm{wt} \%$ solution of HPC. Plots for medium are also given. Concentrations of suspensions are expressed by the sum of weights, 40 wt $\%$ HPC plus wt $\% \mathrm{TiO}_{2}$. 
disperse particles and differ essentially from the dynamic properties of 45 and $50 \mathrm{wt} \%$ HPC solutions shown in Figs. 5 and 6. This means that the interaction between HPC and $\mathrm{TiO}_{2}$ is minor, if any. Only the curves of $60 \mathrm{wt} \%$ suspension seem similar at first glance to those of liquid crystals in that they tend to flatten at the low frequency region, but a distinct difference is found when the curves are compared all over the frequency region covered. While both $G^{\prime}$ and $G^{\prime \prime}$ of suspensions are larger than those of $40 \mathrm{wt} \% \mathrm{HPC}$ solution throughout the entire region of frequency, the curves of $G^{\prime}$ and $G^{\prime \prime}$ of liquid crystals in the high frequency region are situated at lower positions than those of 39 and $41 \mathrm{wt} \%$ HPC solutions, as Figs. 5 and 6 show.

In Fig. 12 is shown the plot of absolute value of complex viscosity against angular frequency in the logarithmic scale. The complex viscosities of suspensions are larger than that of the medium throughout the whole frequency region covered and increase regularly with concentration. This relationship is fundamentally different from that of the dynamic viscosities of liquid crystals. It has been shown in Fig. 8 that the liquid crystals having specific internal structures show rather the lower viscosities than the structureless isotropic solutions. Furthermore, the order of viscosity of liquid crystal is not in accord with the order of concentration. Similar relationship has been recognized also in the steady-flow viscosities of liquid crystals, as given in Fig. 10. It is obvious, therefore, that the anomalous behavior of suspensions has its origin fundamentally distinct from the origin from which the unique properties of liquid crystals arise.

The substantial difference of suspensions from liquid crystals is found in the nonlinear viscoelastic behavior of suspensions. Fig. 13 shows the automatic recordings of the input sinusoidal strain $(\gamma)$ and the output torque $(M)$ for 60 and $70 \mathrm{wt} \%$ suspensions at a frequency of $0.1 \mathrm{radian} \cdot \mathrm{sec}^{-1}$. It can be seen in the recordings that the curves of torque are considerably distorted, contrary to the curve of the cholesteric liquid crystal of HPC with a concentration of 60 wt $\%$ shown in Fig. 2. This result indicates that the viscoelastic behavior of suspen-

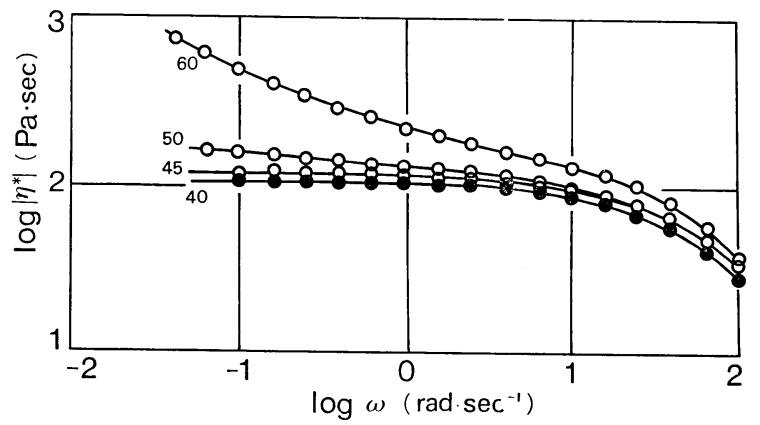

Fig. 12 Logarithmic plot of absolute value of complex viscosity against angular frequency for 45,50 and $60 \mathrm{wt} \%$ suspensions and medium of suspension (40 wt $\%$ solution of HPC).

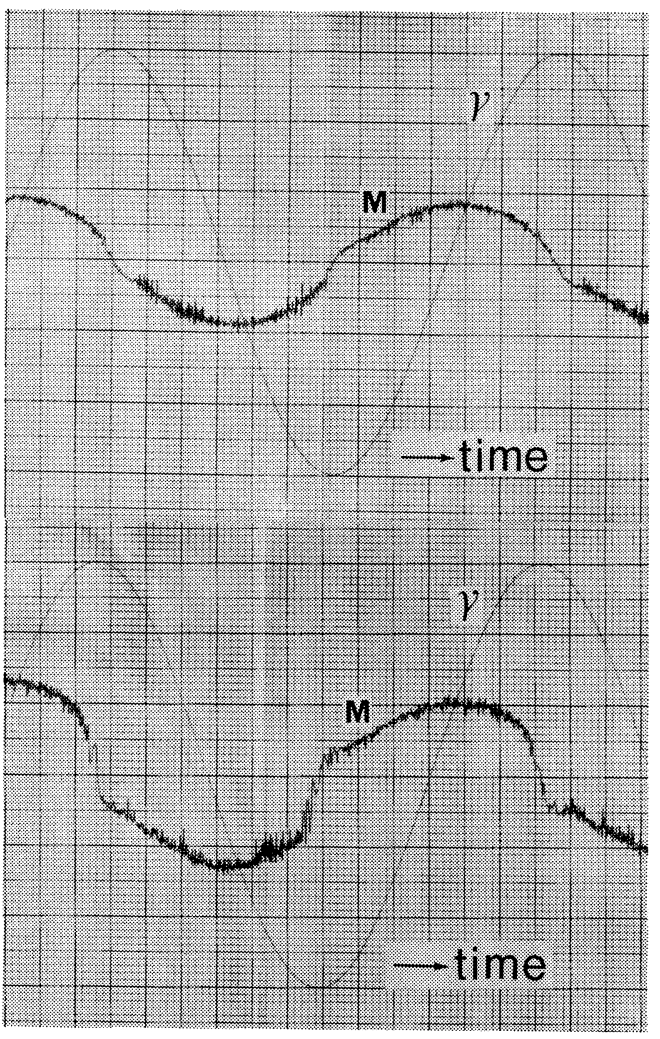

Fig. 13 Automatic recording of input sinusoidal strain $(\gamma)$ and output torque $(M)$ for 60 and $70 \mathrm{wt} \%$ suspensions of $\mathrm{TiO}_{2}$ in $40 \mathrm{wt} \%$ solution of $\mathrm{HPC}$. Upper : 60 wt $\%$, Lower : 70 wt $\%$.

sions is typically nonlinear and fundamentally different from the viscoelastic behavior of liquid crystals which can be regarded as linear. The nonlinear viscoelasticity has been demonstrated in other suspension systems $\left.\left.s^{13}, 15\right), 18\right) \sim 20$ ).

\section{Stress Relaxation}

The anomalous dynamic properties of liquid crystals in the low frequency region suggest the participation of slow relaxation mechanisms in the viscoelastic behavior. The cholesteric liquid crystalline solution of HPC with a concentration of $60 \mathrm{wt} \%$ and the optically isotropic solution with a concentration of 40 wt $\%$ serving as a control were subjected to the steady shear by the rotation of cone with a constant speed to effect the steady flow between cone and plate at shear rates of 1 and 0.1 $\mathrm{sec}^{-1}$. After the steady state had been reached, the motion of cone was stopped abruptly and the change in stress with time was recorded on a graph. Fig. 14 is the automatic recording for the optically isotropic HPC solution. As can be seen in the graph, the relaxation takes place too rapidly to plot the stress against time. On the other hand, the relaxation takes place much more slowly for the liquid crystalline solution whose automatic recording of stress relaxation and the plot of stress against time are shown in Figs. 15 and 16, respectively.

The process of relaxation shown in Fig. 16 can be divided broadly in two periods. In the latter period of relaxation, from about $20 \mathrm{sec}$ downward after the cessation of steady-shear 


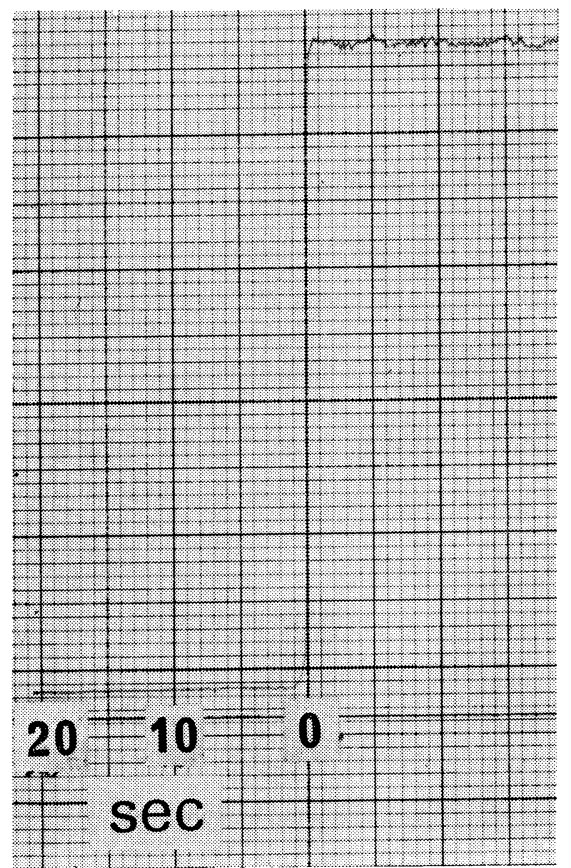

Fig. 14 Automatic recording of stress relaxation after cessation of steady-shear flow at shear rate of 1 $\mathrm{sec}^{-1}$ for $40 \mathrm{wt} \%$ solution of HPC.

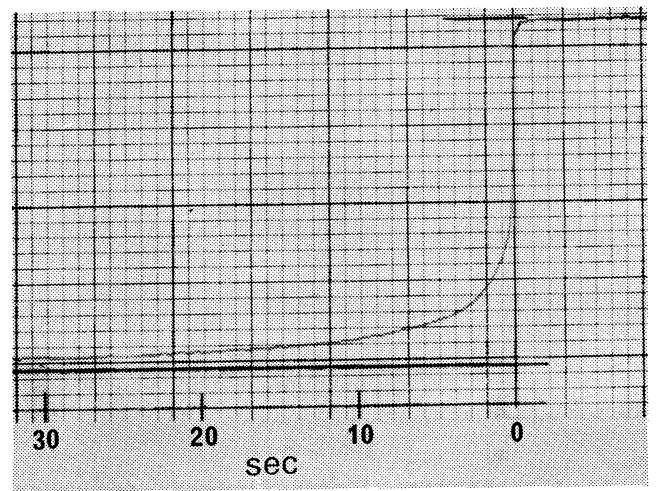

Fig. 15 Automatic recording of stress relaxation after cessation of steady-shear flow at shear rate of 1 $\mathrm{sec}^{-1}$ for $60 \mathrm{wt} \%$ solution of HPC.

flow at a shear rate of $1 \mathrm{sec}^{-1}$, the process is expressed by a simple Maxwell model with a relaxation time of $42 \mathrm{sec}$. For the stress relaxation taking place after the steady flow at a shear rate of $0.1 \mathrm{sec}^{-1}$ also, almost the same relaxation time is measured. This long relaxation time may be related to the recovery of the cholesteric structure from the disturbed state. In the initial stage of relaxation process up to about $20 \mathrm{sec}$ after the cessation of steady flow, a number of mechanisms are complicatedly participated. It can be seen in Fig. 15 that about $90 \%$ of the stress is relaxed within a period of 5 sec.

It is interesting now to relate the stress relaxation with the microscopic observations. ${ }^{5)}$ When the liquid crystalline solution of HPC with a concentration of 48 wt $\%$ is placed between two slides under a polarizing microscope with crossed polars and subjected to shear by the movement of the upper slide, a

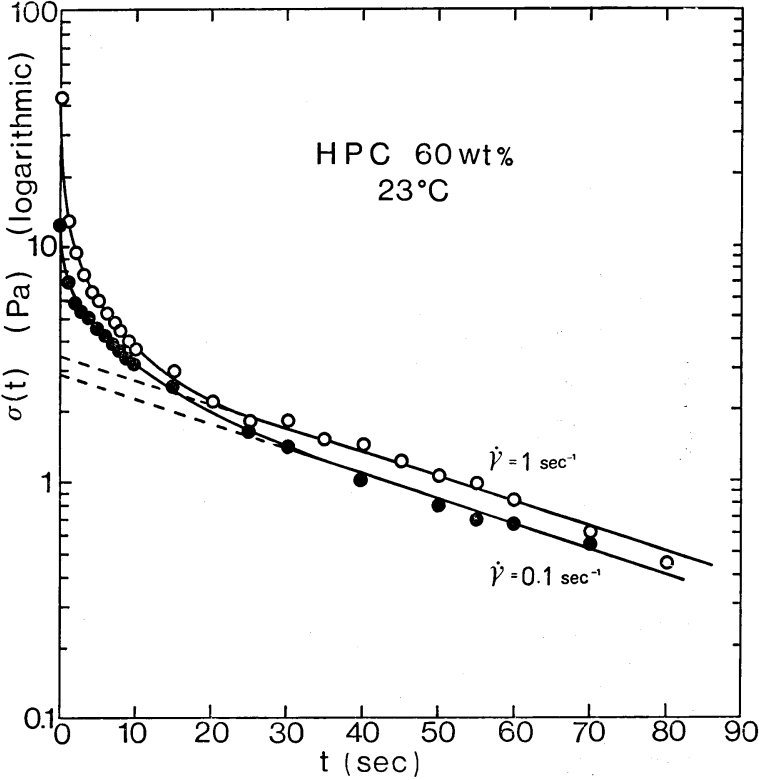

Fig. 16 Logarithm of stress plotted against time after cessation of steady-state flow at shear rates of 0.1 and $1 \mathrm{sec}^{-1}$ for $60 \mathrm{wt} \%$ solution of HPC.

distinct birefringence is observed. This indicates that the aggregates of molecules are arranged uniaxially while the solution flows steadily. When the movement of slide stops abruptly, the uniaxially stretched molecular aggregates relax quickly and shrink to form the zigzag-shaped fibrils. The zigzag structure is demonstrated by the parallel striae running perpendicular to the flow direction which appear within a few seconds after the cessation of flow. The photograph at the top of Fig. 17 shows the striae observed within $5 \mathrm{sec}$ after the cessation of flow. The regularly formed zigzag structure is disordered with time by the thermal effect until a completely random structure is produced, as the middle and bottom photographs in Fig. 17 show. The striated pattern can be demostrated likewise with the highly concentrated solutions of HPC which show the cholesteric texture in the quiescent steady state. It is interesting to see that the initial stage of stress relaxation of liquid crystals in general is related to the formation of the zigzag-shaped fibrillar structure.

\section{Effect of Phase Transition on Dynamic Properties}

The cholesteric phase of HPC is converted readily to the nematic phase by the steady-shear deformation. When the system is released from the shear, the reverse change to the cholesteric phase begins to start immediately. The steady-shear viscosities of 50 and $60 \mathrm{wt} \%$ solutions of HPC are lowered rapidly with increasing shear rate, especially in the region of very small shear rates (See Fig. 10). This effect, as descibed before, is explained in terms of the phase transition from cholesteric to nematic. The next step of phase transition that proceeds automatically from the nematic to the cholesteric type of molecular aggregation was investigated by the measurements 


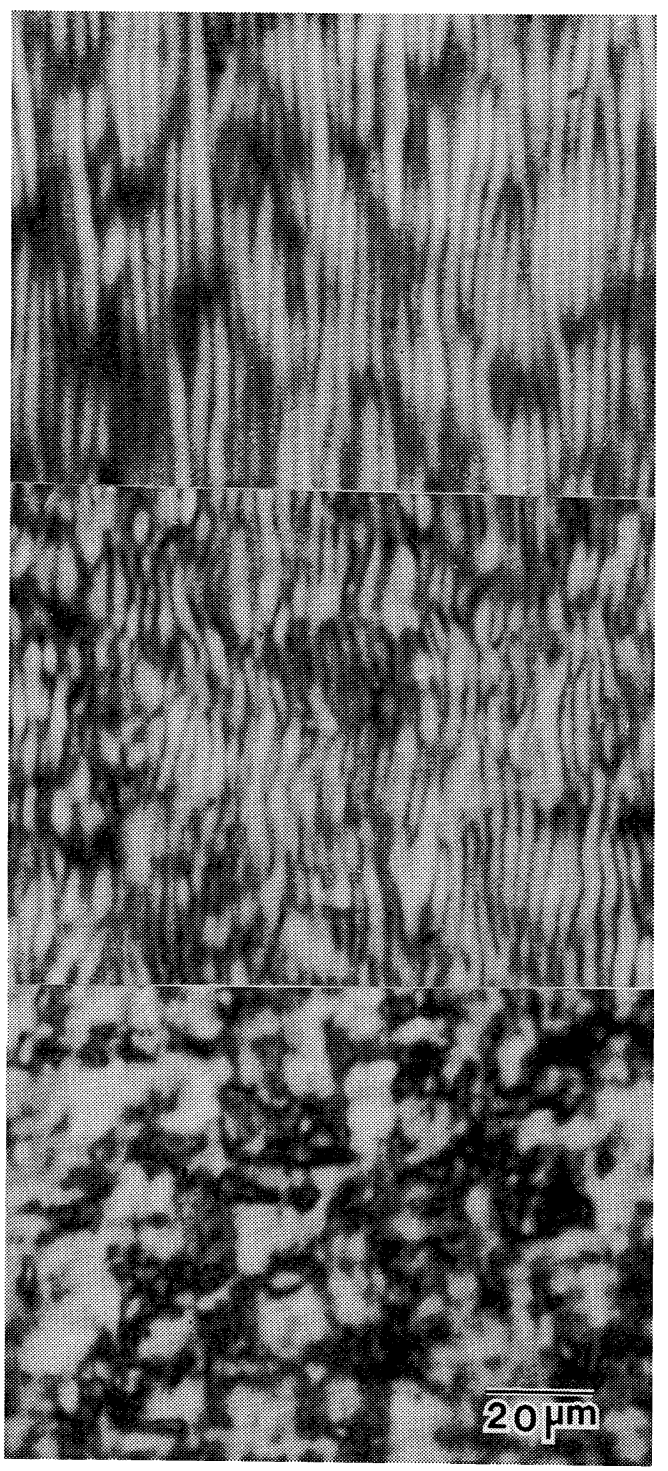

Fig. 17 Striated pattern of $48 \mathrm{wt} \%$ solution of HPC appeaing after cessation of steady flow. Time after cessation of flow : top $5 \mathrm{sec}$, middle $1 \mathrm{~min}$, bottom $4 \mathrm{~min}$.

of dynamic storage modulus.

The experiments were performed by the combination of tests in the following order : dynamic-steady shear-dynamic. The first dynamic tests were made to make sure of the stability of samples to the repeated measurements of dynamic modulus. The results obtained at a frequency of 3 radian $\cdot \mathrm{sec}^{-1}$ are shown in Fig. 18 by open circles and triangles. It can be seen that the values of $G^{\prime}$ of all samples measured at regular intervals of time are almost constant. This indicates that all of the samples used remain unaffected by the repetition of intermittent oscillatory deformation.

The first dynamic test of each sample was followed by the steady shear experiment which was conducted by the stationary rotation of cone in contact with the sample. The shear rate was $3 \mathrm{sec}^{-1}$. The steady-shear flow continued for $2 \mathrm{~min}$ for

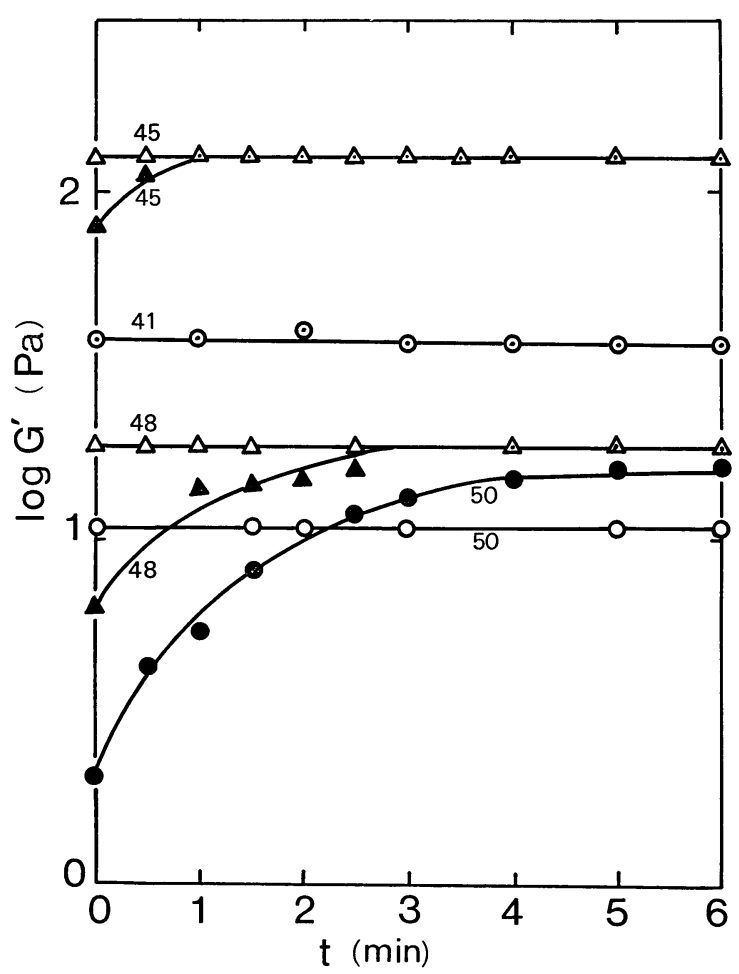

Fig. 18 Plot of $\log G^{\prime}$ against time in combined tests performed in the order: dynamic-steady sheardynamic. Angular frequency at dynamic tests, 3 radian- $\sec ^{-1}$. Rate of shear at steady-shear tests, $3 \sec ^{-1}$.

$\bigcirc, \triangle:$ Dynamic measurements before steady-shear flow.

- $\mathbf{\Delta}$ : Dynamic measurements after steady-shear flow.

attaining the equilibrium. After a short intermission of $20 \mathrm{sec}$ from the cessation of the steady flow, the dynamic storage modulus was measured intermittently with intervals of $30 \mathrm{sec}$ at an angular frequency of $3 \mathrm{radian} \cdot \mathrm{sec}^{-1}$. The results are shown by solid circles and triangles in Fig. 18 which illustrates the plot of $\log G^{\prime}$ against time.

The solution of HPC is influenced by the steady shear in different manner according to the state of solution. The effect of steady shear on the optically isotropic solution with a HPC content of $41 \mathrm{wt} \%$ is confined to the period while the solution is flowing. When released from the shear, the solution soon regains the former state before the flow, because molecules existing separately can move swiftly. As a result, the value of $G^{\prime}$ measured immediately after the cessation of flow is identical with the value measured before flowing.

The optically anisotropic solutions with HPC contents of 45 and 48 wt $\%$ behave in somewhat different manner. The uniaxial orientation of crystalline domains induced by flow remains as it was for a short period of time after the cessation of flow, because the crystalline domains made up of a large number of macromolecules move only slowly. Therefore, a considerably small value of $G^{\prime}$ is observed soon after the flow, 
but as the networks increase in the system, $G^{\prime}$ increases with time up to the former value which the solution showed before undergoing steady flow. It must be noticed in Fig. 18 that a considerably long time which is of the order of several minutes is needed for the reproduction of the original state of solution. The long relaxation time is an important characteristic property of the liquid crystal, as described in the preceding section.

Characteristically different is the behavior of cholesteric phase of HPC solution with a concentration of $50 \mathrm{wt} \%$ or above. The low value of $G^{\prime}$ observed soon after the stop of flow is due to the nematic phase produced by the action of steady-state flow. As time goes on, the system shifts to the cholesteric phase, as manifested in the increase in $G^{\prime}$. Of importance, however, is the fact that the value of $G^{\prime}$ surpasses the original value of solution before undergoing the steady-shear flow. This means that the cho'esteric phase developed in the solution after the cessation of the steady-shear flow is not the same as the cholesteric phase that previously predominated in the system. Asada et al. ${ }^{23)}$ demonstrate the similar effect in the spectra of transmitted light from the cholesteric phase of HPC in the steady state. The spectrum of the solution that has been subjected to a high shear rate shows on standing a higher peak than the spectrum of the same solution before

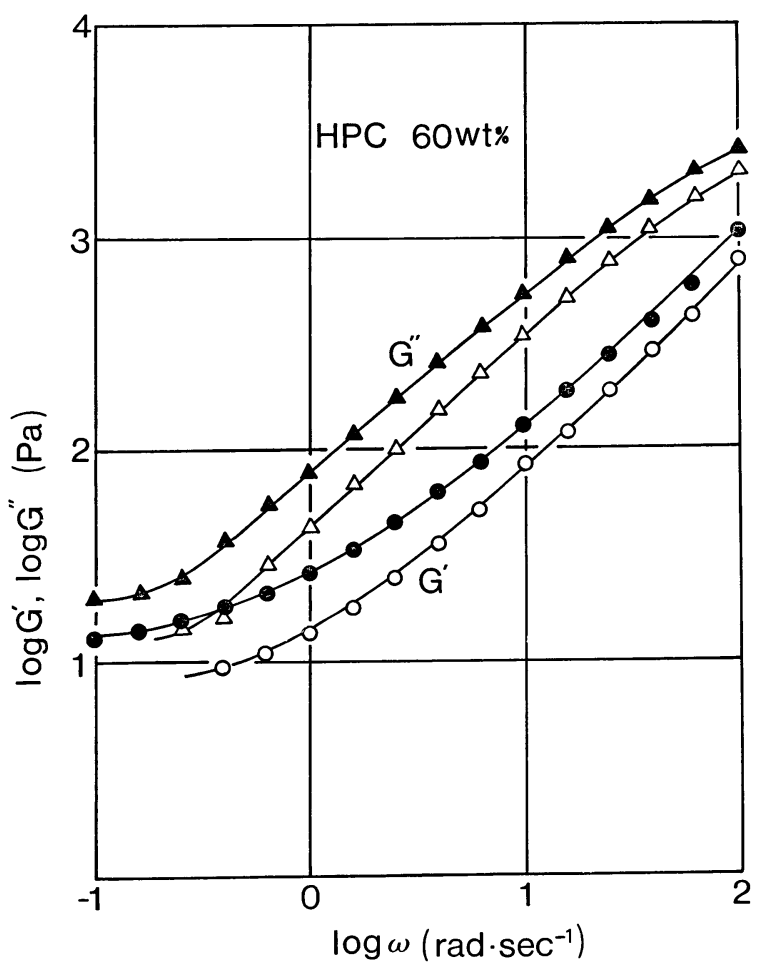

Fig. 19 Plots of $\log G^{\prime}$ and $\log G^{\prime \prime}$ against $\log \omega$ for 60 wt $\%$ solution of HPC in combined tests performed in the order, dynamic-steady shear-dynamic. Rate of shear at steady-shear flow, $1 \mathrm{sec}^{-1}$.

$\bigcirc, \triangle:$ Dynamic measurements before steady-shear flow.

- $\mathbf{\Delta}$ : Dynamic measurements after steady-shear flow. undergoing the shear.

The difference between the dynamic properties measured before and after the steady-shear flow can be demonstrated by another example of cholesteric liquid crystal with a HPC content of $60 \mathrm{wt} \%$. The dynamic storage and loss moduli of the solution were measured in a frequency region from $10^{-1}$ to $10^{2}$ radian $\cdot \mathrm{sec}^{-1}$. Then, the solution was subiected to the steady-shear flow at a shear rate of $1 \mathrm{sec}^{-1}$ for $2 \mathrm{~min}$. The flow was stopped and the solution was allowed to stand for 2 min. Thereon, the dynamic measurement started again with a frequency of $10^{-1}$ radian $\cdot \mathrm{sec}^{-1}$ and continued with regular intervals of $\log \omega$ to reach a final frequency of $10^{2} \mathrm{radian} \cdot \mathrm{sec}^{-1}$. Fig. 19 shows the logarithmic plots of $G^{\prime}$ and $G^{\prime \prime}$ before and after the steady-shear flow against angular frequency. It can be seen that the values of $G^{\prime}$ and $G^{\prime \prime}$ measured after the steady-shear flow are evidently larger than those measured before the flow throughout the whole region of frequency. This series of experiments indicates that the structure of a definite cholesteric liquid crystal in the steady state is not an inherent character native to the system but is largely dependent on the history of mechanical treatments to which the system has been subjected thus far.

The authors thank to Mr. Shozo Murakami of Institute for Chemical Research of Kyoto University for his valuable technical assistance.

(Presented at the 31st Rheology Symposium in Ube, Nov. 8, 1983)

\section{REFERENCES}

1) R.S. Porter and J.F. Johnson, Rheology, F.R. Eirich, Vol. 4, Chapter 9, Academic Press, New York (1967).

2) T. Asada and S. Onogi, Polymer Eng. Reviews, 3, 323 (1983).

3) M. Horio, T. Asada and S. Onogi, Abstract of Papers of 179th American Chemical Society National Meeting at Houston, Texas, March 1980. Symposium on Liquid Crystal Formation in Cellulose Derivatives, Cellulose, Paper and Textile Chemistry Division.

4) M. Horio, Ann. Rep. Res. Inst. Chemical Fibers, Japan, 38, 79 (1981).

5) M. Horio, S. Ishikawa and K. Oda, U.S.-Japan Seminar on Polymer Liquid Crystals, Reprints, p. 218 (1983).

6) F.F. -Ho, R.R. Kohler and G.A. Ward, Anal. Chem., 44, 178 (1972).

7) K. Kimura, T. Shigemura, M. Kubo and Y. Maru, Makromol. Chem., 186, 61 (1985).

8) M.W. Tschoegl and J.D. Ferry, J. Phys. Chem., 86, 1474 (1964).

9) J.D. Ferry, Viscoelastic Properties of Polymers, 3rd Ed., Chapters 9, 17, John Wiley and Sons, Inc., New York (1980). 
10) S. Onogi, T. Masuda and T. Matsumoto, Nippon Kagaku Zasshi, 88, 854 (1967).

11) S. Onogi, T. Masuda and T. Matsumoto, Ann. Rep. Res. Inst. Chemical Fibers, Japan, 25, 15 (1968).

12) S. Onogi, T. Masuda and T. Matsumoto, Nippon Kagaku Zasshi, 89, 464 (1968).

13) T. Matsumoto, T. Masuda, T. Tsutsui and S. Onogi, Nippon Kagaku Zasshi, 90, 360 (1970).

14) S. Onogi, T. Masuda and T. Matsumoto, Trans. Soc. Rheology, 14, 275 (1970).

15) S. Onogi, T. Masuda and T. Matsumoto, J. Soc. Materials Sci., Japan, 19, 341 (1970).

16) S. Onogi, T. Matsumoto, Shikizai, 43, 389 (1970).
17) T. Matsumoto, Y. Segawa, Y. Warashina and S. Onogi, J. Soc. Materials Sci., Japan, 21, 419 (1972).

18) T. Matsumoto, C. Hitomi and S. Onogi, J. Soc. Rheol., Japan, 2, 12 (1974).

19) S. Onogi and T. Matsumoto, Polymer Eng. Reviews, 1, 45 (1981).

20) K. Umeya, T. Isoda and Y. Otsubo, J. Soc. Rheol., Japan, 3, 7 (1975).

21) T. Amari and K. Watanabe, Polymer Eng. Reviews, 3, 277 (1983).

22) T. Asada, K. Toda and S. Onogi, Mol. Cryst. Liq. Cryst., 68, 231 (1981).

23) T. Asada, Private communication. 